J. Korean Math. Soc. 49 (2012), No. 4, pp. 855-865

http://dx.doi.org/10.4134/JKMS.2012.49.4.855

\title{
COMMUTING POWERS AND EXTERIOR DEGREE OF FINITE GROUPS
}

\author{
Peyman Niroomand, Rashid Rezaei, and Francesco G. Russo
}

\begin{abstract}
Recently, we have introduced a group invariant, which is related to the number of elements $x$ and $y$ of a finite group $G$ such that $x \wedge y=1_{G \wedge G}$ in the exterior square $G \wedge G$ of $G$. This number gives restrictions on the Schur multiplier of $G$ and, consequently, large classes of groups can be described. In the present paper we generalize the previous investigations on the topic, focusing on the number of elements of the form $h^{m} \wedge k$ of $H \wedge K$ such that $h^{m} \wedge k=1_{H \wedge K}$, where $m \geq 1$ and $H$ and $K$ are arbitrary subgroups of $G$.
\end{abstract}

\section{Homological algebra and commutativity degree}

All the groups, which are considered in the present paper, are supposed to be finite. Classical contributions of Brown, Ellis, Johnson, Loday and Robertson $[2,3,6]$ and the recent contributions $[12,10,11]$ contain technical notions of homological algebra which generalize the well-known Schur multiplier of a group to the Schur multiplier of a pair of groups and to the Schur multiplier of a triple of groups. By a triple $(G, H, K)$ we mean a group $G$ with two normal subgroups $H$ and $K$ and the Schur multiplier of $(G, H, K)$ is an abelian group $M(G, H, K)$, defined in terms of the mapping cone $B(G, H, K)$ of the canonical cofibration $B(G, K) \rightarrow B(G / K, H K / H)$. The notions of mapping cone and of canonical cofibration are commonly used in algebraic topology and they can be found in [6]. An alternative way, which we prefer in this context, is to relate the Schur multiplier of a triple with an important construction in algebraic topology and group theory.

A group $G$ acts by conjugation on its normal subgroups $H$ and $K$ via the rule ${ }^{g} x=g x g^{-1}$ for $g$ in $G$ and $x$ in $H$ or $K$, and the exterior product $H \wedge K$ is defined as the group generated by the symbols $h \otimes k$, subject to the relations:

$$
h h^{\prime} \otimes k=\left({ }^{h} h^{\prime} \otimes{ }^{h} k\right)(h \otimes k), \quad k k^{\prime} \otimes h=(k \otimes h)\left({ }^{k} h \otimes{ }^{k} k^{\prime}\right), \quad y \otimes y=1,
$$

Received June 19, 2011; Revised November 23, 2011.

2010 Mathematics Subject Classification. Primary 20J99, 20D15; Secondary 20D60, $20 \mathrm{C} 25$.

Key words and phrases. $m$-th relative exterior degree, commutativity degree, exterior product, Schur multiplier, homological algebra. 
where $h, h^{\prime} \in H, k, k^{\prime} \in K$ and $y \in H \cap K$. Briefly, $h \wedge k$ denotes $h \otimes k$ satisfying all the above relations. The map

$$
\kappa^{\prime}: h \wedge k \in H \wedge K \mapsto[h, k]=h k h^{-1} k^{-1} \in[H, K]
$$

turns out to be a group epimorphism, whose kernel ker $\kappa^{\prime}$ is abelian. In [6] it is shown

$$
\text { ker } \kappa^{\prime} \simeq M(G, H, K)
$$

whenever $G=H K$ and this is a very useful way to look at $M(G, H, K)$.

Omitting the relation $y \otimes y=1$, we may define the non-abelian tensor product $H \otimes K$ of $H$ and $K$. By analogy, the map

$$
\kappa: h \otimes k \in H \otimes K \mapsto[h, k]=h k h^{-1} k^{-1} \in[H, K]
$$

turns out to be a group epimorphism, whose kernel

$$
\text { ker } \kappa=J(G, H, K)
$$

is again abelian. We note that $J(G, H, K)$ is related to the fundamental group of a covering space and has significant interest in algebraic topology (see $[2,3$, $6]$ ). We note that $G=H K$ (with $H$ and $K$ normal in $G$ ) satisfies the following commutative diagram

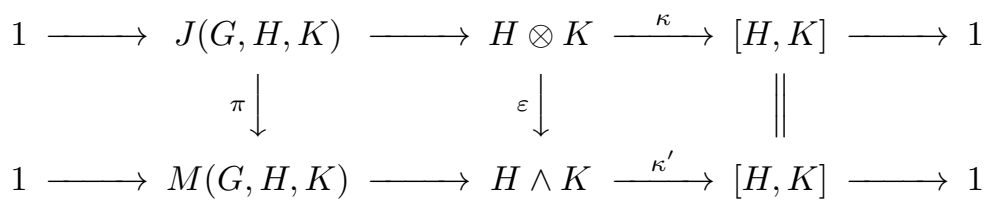

with central extensions as rows and natural epimorphisms

$$
\begin{gathered}
\pi: h \otimes k \in J(G, H, K) \mapsto h \wedge k \in M(G, H, K), \\
\varepsilon: h \otimes k \in H \otimes K \mapsto h \wedge k \in H \wedge K
\end{gathered}
$$

as columns. Of course, if $G=H=K$, then $M(G)$ is the Schur multiplier of $G, H \otimes K=G \otimes G$ is the non-abelian tensor square of $G$ and, in particular, $G^{a b} \otimes_{\mathbb{Z}} G^{a b}$ is the usual tensor square of an abelian group.

It may be helpful to recall that the actions of $H$ on $K$ induce an action

$$
a \in H * K \longmapsto{ }^{a}(h \otimes k)={ }^{a} h \otimes{ }^{a} k \in H \otimes K,
$$

which allows us to see $H \otimes K$ as a suitable homomorphic image of the central product $H * K$. When we have actions, there are stabilisers and orbits. We are going to focuse our attention on a specific action. If $x \in G$, the exterior centralizer of $x$ in $G$ is the set

$$
C_{G}^{\wedge}(x)=\left\{a \in G \mid a \wedge x=1_{G \wedge G}\right\},
$$

which turns out to be a subgroup of $G$ and the exterior center of $G$ is the set

$$
Z^{\wedge}(G)=\left\{g \in G \mid 1_{G \wedge G}=g \wedge y \in G \wedge G, \forall y \in G\right\}=\bigcap_{x \in G} C_{G}^{\wedge}(x)
$$


which is a subgroup of the center $Z(G)$ of $G$. Further details can be found in $[11,12]$. Very briefly, we mention that the interest in studying $C_{G}^{\wedge}(x)$ and $Z^{\wedge}(G)$ is due to the fact that they allow us to decide whether $G$ is a capable group or not, that is, whether $G$ is isomorphic to $E / Z(E)$ for some group $E$ or not. [1] illustrates that capable groups are well-known and classified.

Now we recall from $[8,9]$ that the commutativity degree of $G$ is the ratio

$$
d(G)=\frac{|\{(x, y) \in G \times G \mid[x, y]=1\}|}{|G|^{2}}=\frac{1}{|G|^{2}} \sum_{x \in G}\left|C_{G}(x)\right|=\frac{k(G)}{|G|},
$$

where $k(G)$ is the number of the $G$-conjugacy classes $[x]_{G}=\left\{x^{g} \mid g \in G\right\}$ that constitute $G$. There is a wide range of results on $d(G)$ and its generalizations in the last decades. For instance, given an arbitrary subgroup $H$ of $G$, in [7] the $n$-th relative nilpotency degree of $G$ was introduced as follows

$$
\begin{aligned}
d^{(n)}(H, G) & =\frac{\left|\left\{\left(h_{1}, \ldots, h_{n}, g\right) \in H^{n} \times G \mid\left[h_{1}, \ldots, h_{n}, g\right]=1\right\}\right|}{|H|^{n}|G|} \\
& =\frac{1}{|H|^{n}|G|} \sum_{h_{1}, \ldots, h_{n} \in H}\left|C_{G}\left(\left[h_{1}, \ldots, h_{n}\right]\right)\right| .
\end{aligned}
$$

It is clearly a generalization of $d(G)$, and, in case $n=1$, the further generalization was proposed

$$
d(H, K)=\frac{|\{(h, k) \in H \times K \mid[h, k]=1\}|}{|H||K|}=\frac{1}{|H||K|} \sum_{h \in H}\left|C_{K}(h)\right|=\frac{k_{K}(H)}{|H|},
$$

where $H$ is a normal subgroup of $G, K$ is an arbitrary subgroup of $G$ and $k_{K}(H)$ is the number of the $K$-conjugacy classes $[h]_{K}=\left\{h^{k} \mid k \in K\right\}$ that constitute $H$.

We will focuse on a recent contribution in [10], where the exterior degree of $G$ can be written as

$$
d^{\wedge}(G)=\frac{\left|\left\{(x, y) \in G \times G \mid x \wedge y=1_{G \wedge G}\right\}\right|}{|G|^{2}},
$$

which can be written by $[10$, Lemma 2.2$]$ as

$$
d^{\wedge}(G)=\frac{1}{|G|} \sum_{i=1}^{k(G)} \frac{\left|C_{G}^{\wedge}\left(x_{i}\right)\right|}{\left|C_{G}\left(x_{i}\right)\right|} .
$$

Analogously, given two arbitrary subgroups $H$ and $K$ of $G$, we define for $m \geq 1$ the $m$-th relative exterior degree of $H$ and $K$ in $G$

$$
d_{m}^{\wedge}(H, K)=\frac{\left|\left\{(h, k) \in H \times K \mid h^{m} \wedge k=1_{H \wedge K}\right\}\right|}{|H||K|} .
$$

In particular, $d_{m}^{\wedge}(G)=d_{m}^{\wedge}(G, G)$ is the $m$-th exterior degree of $G$ and, of course, $d_{1}^{\wedge}(G, G)=d^{\wedge}(G)$ so that it is meaningful to generalize the bounds in [10]. We also note that for $H=G$ and $m=1$ there are results on $d^{\wedge}(G, K)$ in [11]. While the commutativity degree represents the probability that two randomly 
picked elements of $G$ are commuting, the $n$-th relative nilpotency degree is a variation on this theme. By analogy with the operator $\wedge$, the $m$-th relative exterior degree is a variation on the theme of the exterior degree, involving the powers of $x$ and the single element $y$. We will study the effects of $d_{m}^{\wedge}(H, K)$ on the structure of $G$ in the successive sections.

\section{Basic properties}

An immediate observation is that we may rewrite $d_{m}^{\wedge}(H, K)$ as:

$$
d_{m}^{\wedge}(H, K)=\frac{1}{|H||K|} \sum_{h \in H}\left|C_{K}^{\wedge}\left(h^{m}\right)\right| .
$$

Assume that $H$ is normal in $G$ and $C_{1} \ldots, C_{k_{K}(H)}$ are the $K$-conjugacy classes that constitute $H$. It follows that

$$
\begin{aligned}
|H||K| d_{m}^{\wedge}(H, K) & =\sum_{h \in H}\left|C_{K}^{\wedge}\left(h^{m}\right)\right|=\sum_{i=1}^{k_{K}(H)} \sum_{h \in C_{i}}\left|C_{K}^{\wedge}\left(h^{m}\right)\right| \\
& =\sum_{i=1}^{k_{K}(H)}\left|K: C_{K}\left(h_{i}\right)\right|\left|C_{K}^{\wedge}\left(h_{i}^{m}\right)\right| \\
& =\sum_{i=1}^{k_{K}(H)} \frac{|K|}{\left|C_{K}\left(h_{i}^{m}\right)\right|} \frac{\left|C_{K}\left(h_{i}^{m}\right)\right|}{\left|C_{K}\left(h_{i}\right)\right|}\left|C_{K}^{\wedge}\left(h_{i}^{m}\right)\right| \\
& =|K| \sum_{i=1}^{k_{K}(H)}\left(\frac{\left|C_{K}\left(h_{i}^{m}\right)\right|}{\left|C_{K}\left(h_{i}\right)\right|}\right) \frac{\left|C_{K}^{\wedge}\left(h_{i}^{m}\right)\right|}{\left|C_{K}\left(h_{i}^{m}\right)\right|} \\
& =|K| \sum_{i=1}^{k_{K}(H)} \alpha(m, i) \frac{\left|C_{K}^{\wedge}\left(h_{i}^{m}\right)\right|}{\left|C_{K}\left(h_{i}^{m}\right)\right|},
\end{aligned}
$$

where $\alpha(m, i)$ is the index of $\left|C_{K}\left(h_{i}\right)\right|$ in $\left|C_{K}\left(h_{i}^{m}\right)\right|$ and thus a natural number. The assumption that $H$ has to be normal in $G$ is done in order to have an entire conjugacy class which is fixed under the action of $K$ on $H$. For the rest of the paper it may be helpful to define the group

$$
L\left(m, h_{i}, K\right)=\frac{C_{K}\left(h_{i}^{m}\right)}{C_{K}^{\wedge}\left(h_{i}^{m}\right)} .
$$

Lemma 2.1. Let $H$ be a normal subgroup of a group $G$ and $K$ be a subgroup of $G$. Then

$$
d_{m}^{\wedge}(H, K)=\frac{1}{|H|} \sum_{i=1}^{k_{K}(H)} \alpha(m, i) \frac{\left|C_{K}^{\wedge}\left(h_{i}^{m}\right)\right|}{\left|C_{K}\left(h_{i}^{m}\right)\right|}=\frac{1}{|H|} \sum_{i=1}^{k_{K}(H)} \frac{\alpha(m, i)}{\left|L\left(m, h_{i}, K\right)\right|} .
$$

In particular, if $G=H K$ and $K$ is normal in $G$, then $L\left(m, h_{i}, K\right)$ is isomorphic to a subgroup of $M(G, H, K)$. 
Proof. The first part follows from (2). Now assume that $G=H K$ for $H$ and $K$ normal in $G$. The exact sequence (1) implies that for all $i=1, \ldots, k_{K}(H)$ the map $x \in C_{K}\left(h_{i}^{m}\right) \mapsto h_{i}^{m} \wedge x \in M(G, H, K)$ is a homomorphism of groups. On the other hand, its kernel is $C_{K}^{\wedge}\left(h_{i}^{m}\right)$, and, consequently, $L\left(m, h_{i}, K\right)$ is isomorphic to a subgroup of $M(G, H, K)$.

The sequence $d_{m}^{\wedge}(H, K)$ is monotone in the sense of the next result. We must assume that $m$ is a prime power. This will be necessary (but not sufficient) to have the subgroup lattice of a cyclic group which is a chain.

Proposition 2.2. Let $H$ and $K$ be subgroups of a group $G$ and $p$ be a prime divisor of $|H|$. Then there exists an integer $r \geq 1$ such that

$$
d_{p^{r-1}}^{\wedge}(H, K) \geq d_{p^{r-2}}^{\wedge}(H, K) \geq \cdots \geq d_{p}^{\wedge}(H, K) \geq d^{\wedge}(H, K) .
$$

Proof. Let $h \in H$ be of order $p^{r}$ for some integer $r \geq 1$. Then $\{1\}=\left\langle h^{p^{r}}\right\rangle \leq$ $\left\langle h^{p^{r-1}}\right\rangle \leq \cdots \leq\langle h\rangle$ implies $C_{K}^{\wedge}(\{1\})=K \geq C_{K}^{\wedge}\left(h^{p^{r-1}}\right)=C_{K}^{\wedge}\left(\left\langle h^{p^{r-1}}\right\rangle\right) \geq \cdots \geq$ $C_{K}^{\wedge}\left(h^{p}\right)=C_{K}^{\wedge}\left(\left\langle h^{p}\right\rangle\right) \geq C_{K}^{\wedge}(h)=C_{K}^{\wedge}(\langle h\rangle)$. Therefore

$$
\sum_{h \in H}\left|C_{K}^{\wedge}(h)\right| \leq \sum_{h \in H}\left|C_{K}^{\wedge}\left(h^{p}\right)\right| \leq \cdots \leq \sum_{h \in H}\left|C_{K}^{\wedge}\left(h^{p^{r-1}}\right)\right|,
$$

from which we deduce

$$
d^{\wedge}(H, K) \leq d_{p}^{\wedge}(H, K) \leq \cdots \leq d_{p^{r-1}}^{\wedge}(H, K) .
$$

Among groups with trivial Schur multiplier there are important classes of groups. For instance, a cyclic group $C=\langle c\rangle$ has $|M(C)|=1$; a metacyclic group of the form

$$
D=\left\langle a, b \mid a^{p^{n}}=b^{p}=1, b^{-1} a b=a^{1+p^{n-1}}\right\rangle
$$

(where $n \geq 3$ if $p=2$ ) also has $|M(D)|=1$ (see [1, 2]); finally, looking at [4], several sporadic simple groups have trivial Schur multiplier. In our context, we are interested in seeing what happens to $d_{m}^{\wedge}(H, K)$ when $M(G, H, K)$ is trivial. As a consequence of Lemma 2.1, we find immediately the next corollary.

Corollary 2.3. Let $G=H K$ for two normal subgroups $H$ and $K$ of $G$ with $H$ of exponent $p^{r}-1$ for some $r \geq 1$ and some prime $p$. If $M(G, H, K)$ is trivial, then $\alpha\left(p^{r}, i\right)=\left|L\left(p^{r}, h_{i}, K\right)\right|=1$.

Proof. By Lemma 2.1, $\left|L\left(p^{r}, h_{i}, K\right)\right|=1$. Since $H$ has exponent $p^{r}-1$, we get $h_{i}^{p^{r}-1}=1$, that is, $h_{i}^{p^{r}}=h_{i}$ for all $i=1, \ldots, k_{K}(H)$, and so $\alpha\left(p^{r}, i\right)=1$.

When $r \rightarrow \infty$ or $r \rightarrow 0$, Proposition 2.2 yields the following result.

Corollary 2.4. Let $H$ be a normal subgroup of a group $G, K$ a subgroup of $G$ and $p$ a prime divisor of $|H|$. Then

$$
\lim _{r \rightarrow 0} d_{p^{r}}^{\wedge}(H, K)=d^{\wedge}(H, K) .
$$


Furthermore, if $\lim _{r \rightarrow \infty} \frac{\alpha\left(p^{r}, i\right)}{\left|L\left(p^{r}, h_{i}, K\right)\right|}=1$ and the action of $K$ on $H$ induces just one orbit, then

$$
\lim _{r \rightarrow \infty} d_{p^{r}}^{\wedge}(H, K) \leq \frac{1}{p} .
$$

In particular, $d(H, K)=\lim _{r \rightarrow \infty} d_{p^{r}}^{\wedge}(H, K)=\frac{1}{p}$, provided that $|H|=p$.

Proof. The first part of the result follows from Proposition 2.2.

Lemma 2.1 and the assumptions imply

$$
\begin{aligned}
\lim _{r \rightarrow \infty} d_{p^{r}}^{\wedge}(H, K) & =\lim _{r \rightarrow \infty} \frac{1}{|H|} \sum_{i=1}^{k_{K}(H)} \frac{\alpha\left(p^{r}, i\right)}{\left|L\left(p^{r}, h_{i}, K\right)\right|} \\
& =\frac{1}{|H|} \lim _{r \rightarrow \infty} \sum_{i=1}^{k_{K}(H)} \frac{\alpha\left(p^{r}, i\right)}{\left|L\left(p^{r}, h_{i}, K\right)\right|} \\
& =\frac{1}{|H|} \sum_{i=1}^{k_{K}(H)} \lim _{r \rightarrow \infty} \frac{\alpha\left(p^{r}, i\right)}{\left|L\left(p^{r}, h_{i}, K\right)\right|} \\
& =\frac{k_{K}(H)}{|H|}=d(H, K) .
\end{aligned}
$$

The choice of $p$ implies $\frac{1}{|H|} \leq \frac{1}{p}$ and therefore $\lim _{r \rightarrow \infty} d_{p^{r}}^{\wedge}(H, K) \leq \frac{k_{K}(H)}{p}$. In particular, if the action of $K$ on $H$ induces just one orbit, then $k_{K}(H)$ is just one and so $\lim _{r \rightarrow \infty} d_{p^{r}}^{\wedge}(H, K) \leq \frac{1}{p}$. The rest follows clearly from (3).

With respect to direct products there is a sort of natural splitting for $d_{m}^{\wedge}(H$, $K)$ and this is shown below.

Proposition 2.5. If $A, B, C, D$ are subgroups of a group $G$ such that $(|A|,|B|)$ $=(|C|,|D|)=1$, then

$$
d_{m}^{\wedge}(A \times B, C \times D)=d_{m}^{\wedge}(A, C) \cdot d_{m}^{\wedge}(B, D) .
$$

Proof.

$$
\begin{aligned}
& |A \times B||C \times D| d_{m}^{\wedge}(A \times B, C \times D) \\
= & |A||B||C||D| d_{m}^{\wedge}(A \times B, C \times D) \\
= & \sum_{(a, b) \in A \times B}\left|C_{C \times D}^{\wedge}\left(\left(a^{m}, b^{m}\right)\right)\right| \\
= & \left(\sum_{a \in A}\left|C_{C}^{\wedge}\left(a^{m}\right)\right|\right)\left(\sum_{b \in B}\left|C_{D}^{\wedge}\left(b^{m}\right)\right|\right) \\
= & |A||C| d_{m}^{\wedge}(A, C)|B||D| d_{m}^{\wedge}(B, D) .
\end{aligned}
$$

In particular, [10, Lemma 2.10] can be found as a special case of the previous result. Another general property is encountered when we go to form quotients 
and for $m=1$ it can be found in [10, Proposition 2.6]. Before describing it, we introduce the set

$$
Z^{\wedge}(H, K)=\left\{k \in K \mid h \wedge k=1_{H \wedge K} \forall h \in H\right\},
$$

where $H$ and $K$ are normal subgroups of $G$, acting upon each other by conjugation. $Z^{\wedge}(H, K)$ is largely described in [11] when $G=H$ and it is easy to check that $Z^{\wedge}(H, K)$ is a subgroup of $K$, and, in particular, $Z^{\wedge}(G, G)=Z^{\wedge}(G)$.

Proposition 2.6. If $H$ and $K$ are two subgroups of $G$ containing a normal subgroup $N$ of $G$, then

$$
d_{m}^{\wedge}(H, K) \leq d_{m}^{\wedge}(H / N, K / N)
$$

The equality happens, provided $N \subseteq Z^{\wedge}(H, K)$. In the case that $H$ has exponent $m-1$, the converse holds.

Proof.

$$
\begin{aligned}
|H||K| d_{m}^{\wedge}(H, K) & =\sum_{h \in H}\left|C_{K}^{\wedge}\left(h^{m}\right)\right|=\sum_{h N \in H / N} \sum_{n \in N}\left|C_{K}^{\wedge}\left(h^{m} n\right)\right| \\
& =\sum_{h N \in H / N} \sum_{n \in N} \frac{\left|C_{K}^{\wedge}\left(h^{m} n\right) N\right|}{|N|}\left|C_{K}^{\wedge}\left(h^{m} n\right) \cap N\right| \\
& \leq \sum_{h N \in H / N} \sum_{n \in N}\left|C_{K / N}^{\wedge}\left(h^{m} N\right)\right|\left|C_{K}^{\wedge}\left(h^{m} n\right) \cap N\right| \\
& =\sum_{h N \in H / N}\left|C_{K / N}^{\wedge}\left(h^{m} N\right)\right| \sum_{n \in N}\left|C_{K}^{\wedge}\left(h^{m} n\right) \cap N\right| \\
& \leq|N|^{2} \sum_{h N \in H / N}\left|C_{K / N}^{\wedge}\left(h^{m} N\right)\right| \\
& =|H||K| d^{\wedge}(H / N, K / N) .
\end{aligned}
$$

We find always an exact sequence

$$
N \wedge K \stackrel{\varphi}{\longrightarrow} H \wedge K \stackrel{\varepsilon}{\longrightarrow}(H / N) \wedge(K / N) \longrightarrow 1
$$

where $\iota: n \in N \mapsto \iota(n) \in H$ is the natural embedding of $N$ into $H$,

$$
\varphi: n \wedge k \in N \wedge K \mapsto \iota(n) \wedge h \in H \wedge K
$$

and

$$
\varepsilon: h \wedge k \in H \wedge K \mapsto h N \wedge k N \in(H / N) \wedge(K / N)
$$

is induced by the natural epimorphisms of $H$ onto $H / N$ and of $K$ onto $K / N$. If $N \subseteq Z^{\wedge}(H, K)$, then $\operatorname{Im} \varphi=1_{N \wedge K}$ and (4) implies $H / N \wedge K / N \simeq H \wedge K$ so that

$$
\begin{aligned}
& |N|^{2}\left|\left\{(h N, k N) \in H / N \times K / N \mid h^{m} N \wedge k N=1_{(H / N) \wedge(K / N)}\right\}\right| \\
= & \left|\left\{(h, k) \in H \times K \mid h^{m} \wedge k=1_{H \wedge K}\right\}\right|,
\end{aligned}
$$

hence $d_{m}^{\wedge}(H, K)=d_{m}^{\wedge}(H / N, K / N)$. 
Now assume that $d_{m}^{\wedge}(H, K)=d_{m}^{\wedge}(H / N, K / N)$, then from the equality we have $\left|C_{K}^{\wedge}\left(h^{m}\right) \cap N\right|=|N|$ for all $h \in H$, and so $N \subseteq C_{K}^{\wedge}(h)$ for all $h \in H$. It implies that $N \subseteq Z^{\wedge}(H, K)$, as required.

A particular restriction is the following.

Theorem 2.7. Let $G=H K$ for two normal subgroups $H$ and $K$. Then for all $m \geq 1$

$$
\beta(m) \frac{d(H, K)}{|M(G, H, K)|} \leq d_{m}^{\wedge}(H, K) \leq \gamma(m) d(H, K),
$$

where $\beta(m)=\min \left\{\alpha(m, i) \mid i=1, \ldots, k_{K}(H)\right\}$ and $\gamma(m)=\max \{\alpha(m, i) \mid i=$ $\left.1, \ldots, k_{K}(H)\right\}$.

Proof. Keeping in mind Lemma 2.1 and noting that $C_{K}\left(h_{i}^{m}\right) / C_{K}^{\wedge}\left(h_{i}^{m}\right)$ is isomorphic to a subgroup of $M(G, H, K)$, we have

$$
\left|C_{K}^{\wedge}\left(h_{i}^{m}\right)\right| /\left|C_{K}\left(h_{i}^{m}\right)\right| \geq 1 /|M(G, H, K)| .
$$

Therefore

$$
\begin{aligned}
d_{m}^{\wedge}(H, K) & =\frac{1}{|H|} \sum_{i=1}^{k_{K}(H)} \alpha(m, i) \frac{\left|C_{K}^{\wedge}\left(h_{i}^{m}\right)\right|}{\left|C_{K}\left(h_{i}^{m}\right)\right|} \\
& \geq \frac{\beta(m) k_{K}(H)}{|H||M(G, H, K)|} \\
& =\beta(m) \frac{d(H, K)}{|M(G, H, K)|} .
\end{aligned}
$$

On another hand, again Lemma 2.1 implies

$$
d_{m}^{\wedge}(H, K)=\frac{1}{|H|} \sum_{i=1}^{k_{K}(H)} \alpha(m, i) \frac{\left|C_{K}^{\wedge}\left(h_{i}^{m}\right)\right|}{\left|C_{K}\left(h_{i}^{m}\right)\right|} \leq \gamma(m) \frac{k_{K}(H)}{|H|}=\gamma(m) d(H, K)
$$

In $[10,12]$ it was noted that a group $G$ with $Z^{\wedge}(G)=Z(G)$ has strong structural restrictions; among these it was noted in [10] that $d_{1}^{\wedge}(G)=d^{\wedge}(G)=$ $d(G)$. We find something of similar in the next result.

Corollary 2.8. Let $G=H K$ for two normal subgroups $H$ and $K$. If $M(G, H$, $K)$ is trivial and $H$ has exponent $m-1$, then, for all $m \geq 1, d_{m}^{\wedge}(H, K)=$ $d(H, K)$.

Proof. Since $M(G, H, K)=1$ is trivial, the lower bound in (5) is reduced to $\beta(m) d(H, K) . H$ has exponent $m-1$ and then, using the notations of Lemma $2.1, h_{i}^{m-1}=1$, that is, $h_{i}^{m}=h_{i}$ for all $i=1, \ldots, k_{K}(H)$. Consequently, $\alpha(m, i)=\alpha(m)=\beta(m)=\gamma(m)=1$. Then Theorem 2.7 becomes $d(H, K) \leq$ $d_{m}^{\wedge}(H, K) \leq d(H, K)$ and the result follows.

Another consequence of Theorem 2.7 is related to Proposition 2.2. 
Corollary 2.9. Let $G=H K$ for two normal subgroups $H$ and $K$ and $p$ be a prime divisor of $|H|$. Then there exists an integer $r \geq 1$ such that

$\frac{\gamma\left(p^{r-1}\right)}{p} k_{K}(H) \geq d_{p^{r-1}}^{\wedge}(H, K) \geq d_{p^{r-2}}^{\wedge}(H, K) \geq \cdots \geq \beta\left(p^{r-1}\right) \frac{d(H, K)}{|M(G, H, K)|}$.

Proof. It is enough to apply Theorem 2.7 and Proposition 2.2.

In a certain sense, Corollary 2.4 continues to be true without restrictions on $m$. This is illustrated below.

Proposition 2.10. Let $G=H K$ for two normal subgroups $H$ and $K$ such that $[H, K] \neq 1$. Then $d_{m}^{\wedge}(H, K) \leq \gamma(m) \frac{2 p-1}{p^{2}}$, where $p$ is the smallest prime dividing both $|G|$ and $|K|$. In particular, if $H$ has exponent $m-1$, then $d_{m}^{\wedge}(H, K) \leq \frac{2}{p}$.

Proof. From the choice of $p$ we deduce $\left|C_{K}^{\wedge}(H)\right| \leq\left|C_{K}(H)\right| \leq \frac{|K|}{p}$. Now $\left|C_{K}(H)\right|-\left|C_{K}^{\wedge}(H)\right| \leq \frac{|K|}{p}$. The upper bound in Theorem 2.7 and [5, Corollary 3.9] imply

$$
d_{m}^{\wedge}(H, K) \leq \gamma(m) d(H, K) \leq \gamma(m) \frac{2 p-1}{p^{2}} .
$$

In particular, if $H$ has exponent $m-1$, then the argument in Corollary 2.8 implies $\gamma(m)=1$, hence

$$
\frac{2 p-1}{p^{2}}=\frac{2}{p}-\frac{1}{p^{2}} \leq \frac{2}{p} .
$$

The following result justifies the interest for the numerical restrictions on $d_{m}^{\wedge}(H, K)$, which have been the subject of most of the previous bounds. These allow us to describe the position of some subgroups in the whole group, when we consider some special values of the $m$-th relative exterior degree.

Corollary 2.11. Let $H$ be a normal subgroup of $G$ of exponent $m-1$ and $K$ be a normal subgroup of $G$ such that $G=H K$ and $M(G, H, K)$ is trivial. If $d_{m}^{\wedge}(H, K)=\frac{2 p-1}{p^{2}}$ for some prime $p$, then $p$ divides $|G|$. If $p$ is the smallest prime divisor of $|G|$, then $\left|H: C_{H}(K)\right|=\left|K: C_{K}(H)\right|=p$ and, hence, $H \neq K$. In particular, if $d_{m}^{\wedge}(H, K)=\frac{3}{4}$, then $\left|H: C_{H}(K)\right|=\left|K: C_{K}(H)\right|=2$.

Proof. Corollary 2.8 implies $d_{m}^{\wedge}(H, K)=d(H, K)$ for all $m \geq 1$. The rest follows from [5, Proposition 3.1].

\section{Dihedral groups and generalized quaternion groups}

Given the results in Section 2 and those in $[7,8,9,10$, 11], we want to have a closer look at the class of dihedral groups and at that of generalized quaternion groups. As known, these groups possess a cyclic group of index 2 and are metacyclic. We will be quite general and recall that

$$
D_{2 n}=C_{n} \rtimes C_{2}=\left\langle a, b \mid a^{n}=b^{2}=1, b^{-1} a b=a^{-1}\right\rangle
$$


is the dihedral group of order $2 n$, where $n \geq 1$. Assume that $D_{2 n}=\left\{1, a, a^{2}, \ldots\right.$, $\left.\ldots a^{n-1}, b, a b, a^{2} b, \ldots, a^{n-1} b\right\}$ and $t=\operatorname{gcd}(m, n)$. Since $Z^{\wedge}(G)=1$ and $\left(a^{\frac{i n}{t}}\right)^{m}$ $=1$ for $0 \leq i \leq t-1$, for $t$ elements of $D_{2 n}$ we have $\left|C_{D_{2 n}}^{\wedge}\left(x^{m}\right)\right|=2 n$ and for $n-t$ elements $\left|C_{D_{2 n}}^{\wedge}\left(x^{m}\right)\right|=n$. Now, if $m$ is odd, then $\left|C_{D_{2 n}}^{\wedge}\left(\left(a^{j} b\right)^{m}\right)\right|=2$ for $0 \leq j \leq n-1$ and so

$$
d_{m}^{\wedge}\left(D_{2 n}\right)=\frac{n^{2}+n t+2 n}{4 n^{2}} .
$$

If $m$ is even, then $\left(a^{j} b\right)^{m}=1$ and so $\left|C_{D_{2 n}}^{\wedge}\left(\left(a^{j} b\right)^{m}\right)\right|=2 n$, therefore

$$
d_{m}^{\wedge}\left(D_{2 n}\right)=\frac{3 n^{2}+n t}{4 n^{2}}
$$

Summarizing,

$$
d_{m}^{\wedge}\left(D_{2 n}\right)= \begin{cases}\frac{3 n+\operatorname{gcd}(m, n)}{4 n}, & \text { if } m \text { is even } \\ \frac{n+\operatorname{gcd}(m, n)+2}{4 n}, & \text { if } m \text { is odd. }\end{cases}
$$

A similar computation can be made for

$$
Q_{n}=\left\langle a, b \mid a^{n}=b^{2}=(a b)^{2}\right\rangle
$$

which is the generalized quaternion group of order $4 n$. Here we find that

$$
d_{m}^{\wedge}\left(Q_{n}\right)= \begin{cases}\frac{3 n+\operatorname{gcd}(m, n)}{4 n}, & \text { if } m \text { is even, } \\ \frac{n+\operatorname{gcd}(m, n)+2}{4 n}, & \text { if } m \text { is odd. }\end{cases}
$$

From [10, Examples 3.1 and 3.2],

$$
d^{\wedge}\left(D_{2 n}\right)=d\left(D_{2 n}\right)=d^{\wedge}\left(Q_{n}\right)=d\left(Q_{n}\right)
$$

for all $n \geq 1$ and we have just shown that for all $m \geq 1$ (and for all $n \geq 1$ )

$$
d_{m}^{\wedge}\left(D_{2 n}\right)=d_{m}^{\wedge}\left(Q_{n}\right)
$$

We note that $\left|M\left(D_{2 n}\right)\right| \neq 1$ and $\left|M\left(Q_{n}\right)\right|=1$ and so we cannot apply Corollary 2.8 , but (6) and (7) show, in some sense, that the hypothesis of Corollary 2.8 is still true. We note also that (6) and (7) agree with [7, Example 3.11] and $[11$, Section 4].

Acknowledgement. We are grateful to the referee for useful suggestions, which have improved the exposition of the results. The third author wishes also to thank 'Convento La Gancia' in Palermo for hospitality and facilities, during the period in which the present paper has been written. 


\section{References}

[1] F. R. Beyl, U. Felgner, and P. Schmid, On groups occurring as center factor groups, J. Algebra 61 (1979), no. 1, 161-177.

[2] R. Brown, D. L. Johnson, and E. F. Robertson, Some computations of nonabelian tensor products of groups, J. Algebra 111 (1987), no. 1, 177-202.

[3] R. Brown and J.-L. Loday, Van Kampen theorems for diagrams of spaces, Topology 26 (1987), no. 3, 311-335.

[4] J. H. Conway, R. T. Curtis, S. P. Norton, R. A. Parker, and R. A. Wilson, Atlas of Finite Groups, Oxford University Press, Oxford, 1985.

[5] A. K. Das and R. K. Nath, On the generalized relative commutative degree of a finite group, Int. Electr. J. Algebra 7 (2010), 140-151.

[6] G. Ellis, The Schur multiplier of a pair of groups, Appl. Categ. Structures 6 (1998), no. $3,355-371$.

[7] A. Erfanian, P. Lescot, and R. Rezaei, On the relative commutativity degree of a subgroup of a finite group, Comm. Algebra 35 (2007), no. 12, 4183-4197.

[8] P. Lescot, Isoclinism classes and commutativity degrees of finite groups, J. Algebra $\mathbf{1 7 7}$ (1995), no. 3, 847-869.

[9] _ Central extensions and commutativity degree, Comm. Algebra 29 (2001), no. 10, 4451-4460.

[10] P. Niroomand and R. Rezaei, On the exterior degree of finite groups, Comm. Algebra 39 (2011), no. 1, 335-343.

[11] _ The exterior degree of a pair of finite groups, E-print, Cornell University Library, arXiv:1101.4312v1, 2011.

[12] P. Niroomand and F. G. Russo, A note on the exterior centralizer, Arch. Math. (Basel) 93 (2009), no. 6, 505-512.

PEYMAN NiROOMAND

Department of Pure Mathematics

DAMGHAN UNIVERSITY

Damghan P.O. Box 36715-364, Iran

E-mail address: niroomand@du.ac.ir, p_niroomand@yahoo.com

RASHID REZAEI

Department of Mathematics

Malayer University

MALAYER P.O. BOX 657719-95863, IRAN

E-mail address: ras_rezaei@yahoo.com

Francesco G. Russo

Dipartimento di Matematica e Informatica

Universitá di PALERMo

PALERMo 90123, ITALY

AND

Department of Mathematics

Universiti TeKnologi Malaysia

JOHOR BAHRU 81310, MALAYSIA

E-mail address: francescog.russo@yahoo.com 